\title{
Approximate Solution of Volterra-Fredholm Integral Equation with Hilbert Kernel
}

\author{
A.S. Ismail \\ Department of Mathematics, Faculty of Science, Zagazig University, Egypt \\ Temporary address: Mathematics Department, University College in Al-Qunfudah, Umm Al-Qura University, KSA
}

\begin{abstract}
In this work, We use numerical technique to reduce the VolterraFredholm integral equation to a linear system of Fredholm integral equations of the second kind and we apply the product Nystrom method to solve this system of integral equations to get the approximate solution of Volterra-Fredholm integral equation. The results are compared with the exact solution of the integral equation.
\end{abstract}

\section{Keywords:}

Volterra-Fredholm integral equation, Hilbert kernel, product Nystrom method, Numerical treatment.

\section{INTRODUCTION}

Consider the following Volterra-Fredholm integral equation with Hilbert kernel of the form

$$
\begin{gathered}
\phi(x, t)-\frac{1}{2 \pi} \int_{0}^{t} \int_{-\pi}^{\pi} \cot \frac{y-x}{2} \phi(y, \tau) d y d \tau=f(x, t), \\
(x, t) \in]-\pi, \pi[\times[0, T],
\end{gathered}
$$

where $\phi$ is unknown function which is sought in $L_{2}=$ $L_{2}(-\pi, \pi ; 0, T)$ with the usual norm under the condition $\phi( \pm \pi, t)=0$, and $f(x, t) \in L_{2}$ is given function.

Numerical methods for Volterra-Fredholm integral equations have been studied in [3,6-11]. For the linear case, some projection methods are given in [7,8,9]. Kauthen [9] studied continuous time collocation, time discretization collocation methods, and he analyzed their global discrete convergence properties, local and global superconvergence properties. Brunner [3] studied the numerical solution of general integral equations of this type by continuous-time and discrete-time spline collocation methods. The results of Kauthen [9] have been extended to nonlinear Volterra-Fredholm integral equations by Brunner [3]. The Adomian decomposition method for mixed nonlinear Volterra-Fredholm integral equations was presented by K. Maleknejad [10].

\section{THE SYSTEM OF INTEGRAL EQUATIONS}

We use numerical technique [4] to reduce the V-FIE (1) to a linear system of Fredholm integral equations of the second kind. At $t=0$, then Eq.(1) is reduced to

$$
\phi(x, 0)=f(x, 0) .
$$

For $t \neq 0$, we consider the numerical solution of Eq.(1). An obvious numerical procedures is to approximate the integral of Volterra term in Eq.(1) via a quadrature rule which integrates over the variable $\tau$ for a fixed value of $\mathrm{t}$. Thus setting $t=t_{r} \equiv r \ell$, where $\ell=\frac{T}{M}$ is the fixed steplength, we would approximate, in an obvious notation, the integral of Volterra term, in Eq.(1), by

$$
\begin{aligned}
& \int_{0}^{t_{r}} \int_{-\pi}^{\pi} \cot \frac{y-x}{2} \phi(y, \tau) d y d \tau \approx \\
& \ell \sum_{j=0}^{r} \int_{-\pi}^{\pi} u_{r j} \cot \frac{y-x}{2} \phi\left(y, \tau_{j}\right) d y
\end{aligned}
$$

where $\tau_{r}=t_{r}, r=1, \ldots, M$. We note that, the set $\left\{u_{r j}, j=\right.$ $0,1, \ldots, r\}$ represents the weights for an $(r+1)$-point quadrature rule for the interval $[0, r \ell]$. For large $r$ let us consider the simplest possible rule, the repeated Trapezoid rule

$$
\begin{aligned}
& u_{r 0}=u_{r r}=\frac{1}{2}, \quad r=1,2, \cdots, M \\
& u_{r j}=1, \quad j=1,2, \cdots, r-1
\end{aligned}
$$

so that Eq.(3) reduces to the simple form

$$
\begin{gathered}
\int_{0}^{t_{r}} \int_{-\pi}^{\pi} \cot \frac{y-x}{2} \phi(y, \tau) d y d \tau \approx \\
\ell \sum_{j=0}^{r} " \int_{-\pi}^{\pi} \cot \frac{y-x}{2} \phi\left(y, \tau_{j}\right) d y
\end{gathered}
$$

where the double prime on the summation sign implies that the first and the last terms are halved.

This quadrature rule leads to the following set of equations:

$$
\begin{array}{r}
\phi^{(r)}(x)-\frac{\ell}{2 \pi} \sum_{j=0}^{r} " \int_{-\pi}^{\pi} \cot \frac{y-x}{2} \phi^{(j)}(y) d y+E_{r}= \\
f^{(r)}(x), \quad r=1,2, \ldots, M
\end{array}
$$

where $\phi^{(r)}(x)=\phi\left(x,, t_{r}\right), f^{(r)}(x)=f\left(x, t_{r}\right)$ and $E_{r}$ represents the error term in the quadrature rule. If the $E_{r}$ are assumed negligible we can clearly solve this set of equations for $\phi^{(r)}(x)$ by direct forward substitution to obtain

$$
\phi^{(0)}(x)=f^{(0)}(x)
$$


at $r=1$

$$
\phi^{(1)}(x)-\frac{\ell}{4 \pi} \int_{-\pi}^{\pi} \cot \frac{y-x}{2} \phi^{(1)}(y) d y=f^{(1)}(x)+\frac{\ell}{4 \pi} H(x)
$$

where

$$
H(x)=\int_{-\pi}^{\pi} \cot \frac{y-x}{2} f^{(0)}(y) d y .
$$

At $r \geq 2$ we obtain

$$
\phi^{(r)}(x)-\frac{\ell}{4 \pi} \int_{-\pi}^{\pi} \cot \frac{y-x}{2} \phi^{(r)}(y) d y=g^{(r)}(x),
$$

where

$$
\begin{aligned}
g^{(r)}(x)= & f^{(r)}(x)+\sum_{j=1}^{r-1}(-1)^{j} 2\left[f^{(r-j)}(x)-\phi^{(r-j)}(x)\right] \\
& +(-1)^{r+1} \frac{\ell}{4 \pi} H(x), r=2,3, \ldots
\end{aligned}
$$

Equations (8) and (10) represents a system of Fredholm integral equations of the second kind that can be solved by many numerical techniques. In the following section we shall apply the product Nystrom method $[1,2,4]$ to obtain the approximate solutions of Eqs. (8) and (10) which leads to the required approximate solution of the Volterra-Fredholm integral equation (1).

\section{THE PRODUCT NYSTROM METHOD}

According to the product Nystrom method $[1,2,4]$, we approximate the integral term in Eq.s (8) and (10) when $x=x_{i}$ by

$$
\int_{-\pi}^{\pi} \cot \frac{y-x_{i}}{2} \phi^{(r)}(y) d y \approx \sum_{j=0}^{N} w_{i j} \phi^{(r)}\left(y_{j}\right),
$$

where $w_{i j}$ are the weights. Also, we approximate the integral term in (12) by a product integration form of Simpson's rule, we may write

$$
\int_{-\pi}^{\pi} \cot \frac{y-x_{i}}{2} \phi^{(r)}(y) d y=\sum_{j=0}^{\frac{N-2}{2}} \int_{y_{2 j}}^{y_{2 j+2}} \cot \frac{y-x_{i}}{2} \phi^{(r)}(y) d y,
$$

where $x_{i}=y_{i}=-\pi+i h, i=0,1, \ldots, N$ with $h=\frac{2 \pi}{N}$ and $N$ even. Now if we approximate the nonsingular part of the integrand over each interval $\left[y_{2 j}, y_{2 j+2}\right]$ by the second degree Lagrange interpolation polynomial which interpolates it at the points $y_{2 j}, y_{2 j+1}, y_{2 j+2}$, we find

$$
\begin{aligned}
& \int_{-\pi}^{\pi} \cot \frac{y-y_{i}}{2} \phi^{(r)}(y) d y \\
& \approx \sum_{j=0}^{\frac{N-2}{2}} \int_{y_{2 j}}^{y_{2 j+2}} \cot \frac{y-y_{i}}{2}\left\{\frac{\left(y_{2 j+1}-y\right)\left(y_{2 j+2}-y\right)}{2 h^{2}} \phi^{(r)}\left(y_{2 j}\right)\right. \\
& +\frac{\left(y-y_{2 j}\right)\left(y_{2 j+2}-y\right)}{h^{2}} \phi^{(r)}\left(y_{2 j+1}\right) \\
& \left.+\frac{\left(y-y_{2 j}\right)\left(y-y_{2 j+1}\right)}{2 h^{2}} \phi^{(r)}\left(y_{2 j+2}\right)\right\} d y \\
& =\sum_{j=0}^{N} w_{i j} \phi^{(r)}\left(y_{j}\right)
\end{aligned}
$$

where

$$
\begin{aligned}
w_{i, 0} & =\frac{1}{2 h^{2}} \int_{y_{0}}^{y_{2}} \cot \frac{y-y_{i}}{2}\left(y_{1}-y\right)\left(y_{2}-y\right) d y \\
w_{i, 2 j+1} & =\frac{1}{h^{2}} \int_{y_{2 j}}^{y_{2 j+2}} \cot \frac{y-y_{i}}{2}\left(y-y_{2 j}\right)\left(y_{2 j+2}-y\right) d y \\
w_{i, 2 j} & =\frac{1}{2 h^{2}} \int_{y_{2 j-2}}^{y_{2 j}} \cot \frac{y-y_{i}}{2}\left(y-y_{2 j-2}\right)\left(y-y_{2 j-1}\right) d y \\
& +\frac{1}{2 h^{2}} \int_{y_{2 j}}^{y_{2 j+2}} \cot \frac{y-y_{i}}{2}\left(y_{2 j+1}-y\right)\left(y_{2 j+2}-y\right) d y \\
w_{i, N} & =\frac{1}{2 h^{2}} \int_{y_{N-2}}^{y_{N}} \cot \frac{y-y_{i}}{2}\left(y-y_{N-2}\right)\left(y-y_{N-1}\right) d y(14)
\end{aligned}
$$

If we define

$$
\begin{aligned}
\alpha_{j}\left(y_{i}\right) & =\frac{1}{2 h^{2}} \int_{y_{2 j-2}}^{y_{2 j}} \cot \frac{y-y_{i}}{2}\left(y-y_{2 j-2}\right)\left(y-y_{2 j-1}\right) d y, \\
\beta_{j}\left(y_{i}\right) & =\frac{1}{2 h^{2}} \int_{y_{2 j-2}}^{y_{2 j}} \cot \frac{y-y_{i}}{2}\left(y_{2 j-1}-y\right)\left(y_{2 j}-y\right) d y, \\
\gamma_{j}\left(y_{i}\right) & =\frac{1}{2 h^{2}} \int_{y_{2 j-2}}^{y_{2 j}} \cot \frac{y-y_{i}}{2}\left(y-y_{2 j-2}\right)\left(y_{2 j}-y\right) d y,(15)
\end{aligned}
$$

it follows that

$$
\begin{aligned}
w_{i, 0} & =\beta_{1}\left(y_{i}\right), \\
w_{i, 2 j+1} & =2 \gamma_{j+1}\left(y_{i}\right), \\
w_{i, 2 j} & =\alpha_{j}\left(y_{i}\right)+\beta_{j+1}\left(y_{i}\right), \\
w_{i, N} & =\alpha_{\frac{N}{2}}\left(y_{i}\right) .
\end{aligned}
$$

We introduce the change of variable $y=y_{2 j-2}+\zeta h, \quad 0 \leq \zeta \leq 2$, thus the system (15) becomes

$$
\begin{aligned}
& \alpha_{j}\left(y_{i}\right)=\frac{h}{2} \int_{0}^{2} \zeta(\zeta-1) \cot \frac{h(\zeta-i+2 j-2)}{2} d \zeta \\
& \beta_{j}\left(y_{i}\right)=\frac{h}{2} \int_{0}^{2}(1-\zeta)(2-\zeta) \cot \frac{h(\zeta-i+2 j-2)}{2} d \zeta \\
& \gamma_{j}\left(y_{i}\right)=\frac{h}{2} \int_{0}^{2} \zeta(2-\zeta) \cot \frac{h(\zeta-i+2 j-2)}{2} d \zeta
\end{aligned}
$$

After evaluate the previous integrals we can obtain

$$
\begin{aligned}
& w_{i, 2 j+1}=2 z(2-z) \ln \left|\sin \frac{h(2-z)}{2}\right| \\
& -2 z(2-z) \ln \left|\sin \frac{h z}{2}\right| \\
& +4 \sum_{s=0}^{\infty} \frac{(-1)^{s} h^{2 s} B_{2 s}}{(2 s) !}\left(\frac{(2-z)^{1+2 s}(1-z)+z^{1+2 s}(1-z)}{1+2 s}\right. \\
& \left.-\frac{(2-z)^{2+2 s}-z^{2+2 s}}{2(2+2 s)}\right)
\end{aligned}
$$

and

$$
w_{i, 2 j}=6(z-2) \ln \left|\sin \frac{h(2-z)}{2}\right|
$$




$$
\begin{aligned}
& +(z-3)(z-4) \ln \left|\sin \frac{h(4-z)}{2}\right|-z(z-1) \ln \left|\sin \frac{h z}{2}\right| \\
& +\sum_{s=0}^{\infty} \frac{(-1)^{s} h^{2 s} B_{2 s}}{(2 s) !} \times \\
& \left(\frac{6(2-z)^{1+2 s}+z^{1+2 s}(2 z-1)+(4-z)^{1+2 s}(2 z-7)}{1+2 s}\right. \\
& \left.-\frac{2 z^{2+2 s}-2(4-z)^{2+2 s}}{2(2+2 s)}\right)
\end{aligned}
$$

where $B_{2 s}$ are Bernoulli numbers [5], $z=i-2 j+2$ and $1 \leq$ $i, j \leq N-1$. The condition $\phi( \pm \pi, t)=0$, avoids the use of the two points $x= \pm \pi$ and avoids the calculation of $w_{i, 0}$ and $w_{i, N}$. Therefore, the solutions of the integral equations (8) and (10), at every $r$, can be reduced to the solutions of the following system of linear algebraic equations of the form

$$
\phi^{(r)}\left(x_{i}\right)-\frac{\ell}{4 \pi} \sum_{j=1}^{N-1} w_{i j} \phi^{(r)}\left(y_{j}\right)=g^{(r)}\left(x_{i}\right), i=0,1, \ldots, N
$$

or

$$
\Phi^{(r)}-\frac{\ell}{4 \pi} W \Phi^{(r)}=G^{(r)},
$$

which has the solution

$$
\Phi^{(r)}=\left[I-\frac{\ell}{4 \pi} W\right]^{-1} G^{(r)},
$$

where $I$ is the identity matrix and $\operatorname{det}\left(I-\frac{\ell}{4 \pi} W\right) \neq 0$.

\section{NUMERICAL RESULTS}

EXAmple 1. Consider the Volterra-Fredholm integral equation

$$
\phi(x, t)-\frac{1}{2 \pi} \int_{0}^{t} \int_{-\pi}^{\pi} \cot \frac{y-x}{2} \phi(y, \tau) d y d \tau=\sin x .
$$

One can see that $\phi(x, t)=\sin (x+t)$ is the exact solution of $\mathrm{Eq}$ 21]. To achieve the validity, the accuracy and support our theoretical discussion of the proposed method, we notice that approximate solution is nothing but the exact solution as shown in Figure 1 The problem $(21)$ solved numerically with $M=20$ and $T=0.5$ for different values of $N$ to see effect and this is shown in Figure 2 for different values of parameter $t$ in the volterra integral, moreover the parameter $t$ is fixed at $t=t_{i}=i \ell, i=2,9,16$. Clearly as the number of intervals $(N)$ increases, the accuracy of our solution to the integral equation also increases, such a result that should not surprise us.
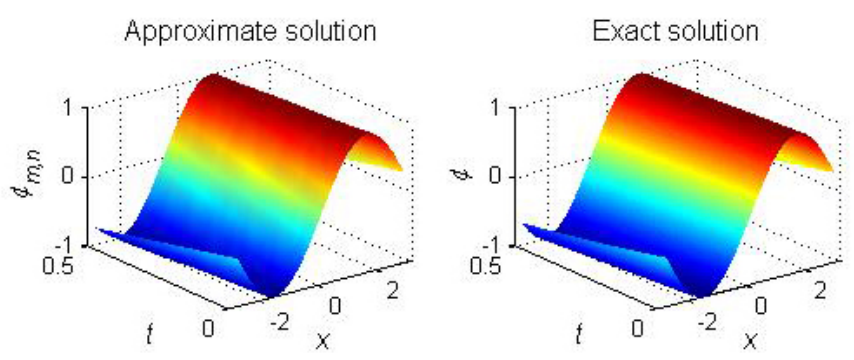

Fig. 1. Approximate solution $\phi_{m n}$ of Eq.(21) with $M=N=20, T=$ 0.5 and the exact solution $\phi(x, t)=\sin (x+t)$.

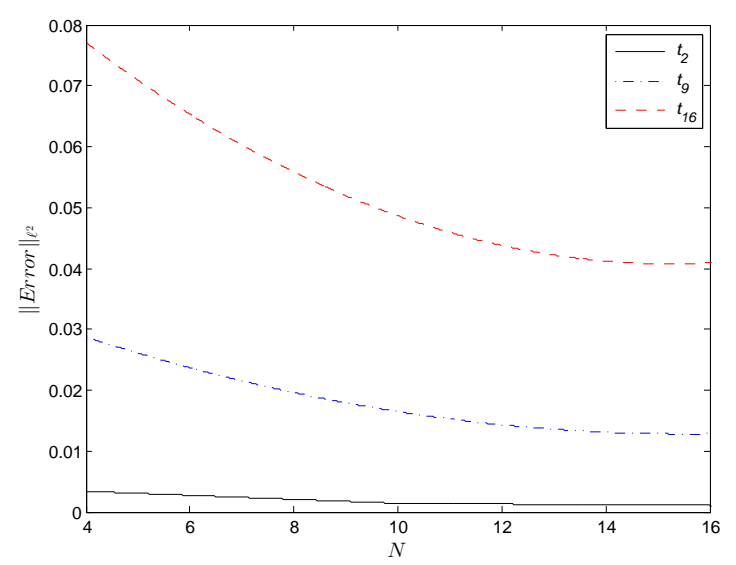

Fig. 2. Norm of the Error for different values of $N$ at fixed points $t_{i}=i \ell$, $i=2,9,16$

\section{REFERENCES}

[1] M.A. Abdou, Khamis I. Mohamed and A.S. Ismail, On the numerical solutions of Fredholm-Volterra integral equation, Appl. Math. Comp. 146, 713-728, (2003).

[2] M.A. Abdou, Khamis I. Mohamed and A.S. Ismail, Toeplitz Matrix and product Nystrom methods for solving the singular integral equation, Le Matematiche LVII-Fasc. I, 21-37, (2002).

[3] H. Brunner, On the numerical solution of nonlinear VolterraFredholm integral equations by collocation methods, SIAM $\mathrm{j}$. Numer. Anal. 27 (4), 987-1000, (1990).

[4] L.M. Delves and J. L. Mohamed, Computational Methods for Integral Equations, Cambridge University Press, (1985).

[5] I.S. Gradshteyn and I.M. Ryzhik, Table of Integrals \& Series and Products, Academic Press, (1980).

[6] H. Guoqiang Asymptotic error expansion for the Nystrom method for a nonlinesr Volterra-Fredholm integral equations, J. Comput. Appl. Math. 59, 49-59, (1995).

[7] H. Guoqiang and Z. Liqing, Asymptotic expansion for the trapezoidal Nystrom method of linesr Volterra-Fredholm integral equations, J. Comput. Appl. Math. 51 (3), 339-348, (1994).

[8] L. Hacia, On approximate solution for integral equations of mixed type, ZAMM. Z. Angew. Math. Mech. 76, 415-416, (1996). 
[9] P.J. Kauthen, Continuous time collocation methods for Volterra-Fredholm integral equations, Numer. Math. 56, 409424, (1989).

[10] K. Maleknejad and M. Hadizadeh, A new computational method for Volterra-Fredholm integral equations, Comp. and Math. with Appl. 37, 1-8, (1999).

[11] B.G. Pachpatte, On mixed Volterra-Fredholm type integral equations, Indian J. Pure Appl. Math. 17, 488-496, (1986). 